\title{
Facial emotion recognition after cosmetic Botulinum toxin injection
}

\author{
Kaveh Shafiei ${ }^{1}$, Behnaz Sedighi², Amir Sherafat ${ }^{3}$ \\ ${ }^{1}$ Neurology Research Center, Shafa Hospital, Kerman University of Medical Sciences, Kerman, Iran \\ ${ }^{2}$ Department of Neurology, Kerman University of Medical sciences, Kerman, Iran \\ ${ }^{3}$ Department of Neurology, Kerman University of Medical sciences, Kerman, Iran
}

\begin{abstract}
Objective. Our understanding of emotions express foster by facial muscle mimicry. By this mechanism, it is hypothesized that cosmetic injections of Botulinum toxin (BTX) into facial muscles and its temporary paralysis of them might impair emotion simulation on the face and lead to attenuate its recognition.

Material and methods. Our study was a before and after study on the subject who came for cosmetic injection of BTX. The Multimodal Emotion Recognition test (MERT) conducted at their sessions: before, at $8^{\text {th }}$ and $20^{\text {th }}$ week after injection. Our primary study endpoint was changing in the MERT's mean score. We also compare the score based on different presented modalities.

Results. 68 volunteers enrolled in our study. The MERT score changed at the $20^{\text {th }}$ week after BTX injection compared to baseline.

Conclusion and discussion. We provide additional arguments in favour of theories asserting that altered facial expression could affect the recognition of emotions.
\end{abstract}

Keywords: facial emotion recognition, Botulinum toxin, multimodal emotion recognition test

\section{INTRODUCTION}

We as human are emotional species. Emotions are the foundation for our proper social interaction. Because of that, our brain continuously involves in perception, recognition and expression of feelings. Most of our non-verbal communications expressed on our faces (1). This also leads to appropriate recognition of the emotion expressed by the others with whom we are communicating and ensures favourable social interactions (2-4). Even Darwin considered this topic in the 19th century. He suggested that the human ability to express and understand emotions is an innate and genetically determined ability and are universal among all human regardless of factors such as social, gender or education (5).

One way that helps us understand the meaning of others feelings is by simulating them in our minds and then trying to imitate them on our face.
It is the basis of so-called the "embodies simulation theory". Based on this theory, our understanding of emotions express foster by facial muscle mimicry $(6,7)$. The tendency to miming the facial emotions expressed by the others, makes a feedback between proprioception and central motor brain networks and help us understand clues displayed by the sender $(8,9)$. In this context, impaired emotion simulation on the face might lead to attenuate its recognition. Interestingly, Even remembering past emotions probably are influenced by how expressed on our face (10). Wiliman James said in 19th century: "Refuse to express a passion, and it dies" (11).

Anger, fear, sadness, disgust, surprise and happiness are six basic emotions which are universal across different cultures and expresse through contractions in facial muscles such a: the Corrugators, Procerus and Frontalis. These muscles have the pivotal role not only in expressing, but also understandings emotions $(2,12)$. Many prominent neu- 
ropsychiatrists, such as Paul Ekman, studied differences in the facial emotion recognition and expression in many different and unrelated cultures around the word from the US to the remote area as New Guinea. Ekman once quoted that: "Can there be emotion without facial expression?" referring the powerful role of facial muscle in emotional expression and recognition (13).

The human's brain uses same neuronal networks and mechanisms in perception, recognition and expression of emotions. It seems that almost all brain regions are involved in facial emotion recognition and expression. Seeing a face activates many regions of our brain. The Calcarian, Fusiform gyrus and Superior temporal lobe are our primary receptive areas. Amygdala, Orbitofrontal, Insular and Cingulate cortex translate that perception to emotional experience and influence the autonomic system outflow. Conceptual knowledge and proper action toward perceived emotion governed by higher cortical area such as prefrontal and somatosensory cortexes. That higher cortical functions enable us to feel empathy towards others emotional experiences and make as what we are as a human (8).

The brain's perceptive and expressive neuronal network work together to improve our understanding of emotion. Whenever we encounter an expressed emotion in others, like fear, disgust or angry, brain regions which control the expression of the same emotion activate in ourself brain. To put it another word, whenever we see someone smile at us, we unconsciously smile or by feeling pain in other faces, the same muscle in our face contract. This simulation helps us to understand and cope better with that expressed emotion.

Aesthetic application of the Botulinum toxin (BTX) into forehead muscle is the most perform cosmetic procedures in the United state and probably around the world. It has few side effects and high patients satisfaction. Localised injections with BTX induces a temporary denervation and paralysis of facial muscles by blocking acetylcholine release at the neuromuscular junction (2). The localised facial muscle weakness could persist for up to 6 months after injection (14). The fact is that facial muscles involved in the simulating emotions like anger, fear, or sadness, are paralysed by this procedure. This effect brings up the question whether by paralysing these muscles, the patient's ability to recognising other facial emotion reduces or not?

A few studies have already shown that there are some neuropsychiatric changes after BTX injection in the facial muscles and even improving mood in depressed patients (14-16). In a randomised clinical trial by M.A. Wollmer in 2012, Depression attenuated in subjects who underwent treatment of glabellar frown lines with BTX (17). David. A, et al. also showed that the cosmetic injection of BTX interrupts emotional language processing (18).

Converting cognitive, purely psychic and subjective human experiences, like emotions, to objective, measurable and neurobiological things is a tremendous neuropsychiatric scientific achievement during past 40 years. Our understanding on this subject has explosively expanded in past few decades by advances in the neuropsychiatric tests (such as the Ekman 360 and the Multimodal Emotion Recognition Test - MERT) and the brain imaging techniques (Functional MRI and Magnetoencephalography (MEG).

The Swiss centre for affective science at the University of Geneva has developed the MERT. During this 45 minutes, test participants asked to choose the emotion presented by actors in different modalities (still picture, video only, audio only and audio-visual). At the end of each session, sum scores calculated for each format as well as a total score for the whole test (expressed in \% correct). In the videos, actors use a standard pseudo-linguistic sentence (i.e., a sentence without meaning) as verbal content (19) (Fig. 1).

It seems that MERT, by using the multimodal platform is more powerful than the previous neuropsychiatric test to detect even minor changes in facial emotion recognition.

Our study aimed to measure the changes in facial emotion recognition ability after BTX injection into facial muscles involved in its expression by MERT. As far as we know, there is few study on changing the emotion recognition changes after the BTX injection and no study has used MERT on this subject.

\section{METHODS}

We conducted a before and after study on patients came for first time cosmetic BTX injection. 

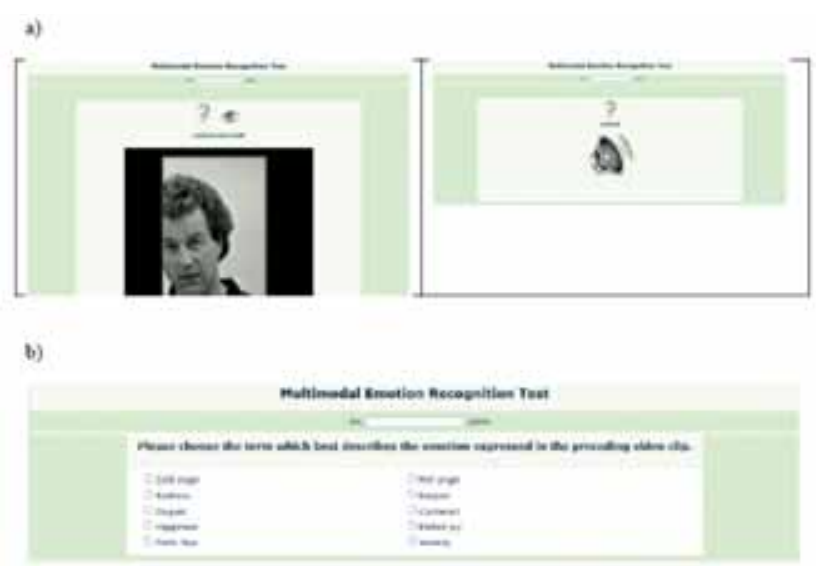

FIGURE 1. Screenshot of the Multimodal emotion recognition test (MERT). a. Audiovisual and audio modalities; $b$. The emotional item to be chosen after stimuli (adapted from MERT user manual)

Interested subjects were informed about our study protocol asked for the history of any psychiatric or neurologic disease and were subjected to a general physical and neurological examination. If no contraindication for the BTX injection existed, they enrolled in our study and a written consent form has been completed. The procedure was free of charges for our subjects.

The ethics committee at the Kerman University of Medical Sciences (KMU), Kerman, Iran approved the study protocol, The study funded by the Neurology research Centre, KMU, independently of any commercial entity.

500 unit of AbobotulinumtoxinA (Dysport, Epsen, $\mathrm{UK}$ ) was dissolved in $0.9 \%$ injectable $\mathrm{NaCl}$ solution at a concentration of $500 \mathrm{U} / 2.5 \mathrm{ml}$.

We injected $20 \mathrm{U}$ to the procerus muscle, $20 \mathrm{U}$ bilaterally to the medial part of the corrugator muscles, and $15 \mathrm{U}$ bilaterally to the lateral portion of the corrugator muscles syringes with $30 \mathrm{G}$ needles. All participants received the injection two weeks after the first experimental session if sufficient targeted muscle paralysis did not induce.

The MERT score calculated at there sessions: before, at $8^{\text {th }}$ and $20^{\text {th }}$ week after injection. Our primary study endpoint was changing in the MERT's mean score. We also compare the score based on different presented modalities.

Data were analysed using two-way analyses of variance (ANOVA) for repeated measures with the factors group (Before, 8 and 20 weeks after injection). Test results with an alpha level 0.05 reported as statistically significant. Statistical analyses were conducted using SPSS 19.0 for Windows.

\section{RESULTS}

68 volunteers enrolled in our study and evaluated by MERT at eight weeks. $82.1 \%$ of the volunteers were female, $89.7 \%$ were between 20 to 60 years old, and $97.1 \%$ had more than 12 years of education. Only 29 subjects returned for 20 weeks evaluation.

Statistical analysis by the Kolmogorov-Smirnov showed that the mean MERT score had a normal distribution.

Fig. 2 shows the mean MERT score before, at 8 and 20 weeks after BTX injection. Subjects had more score when were presented by audiovisual or video only modality and less by still picture or only audio items.

Although there was a reduction in the total MERT score (42.1 to 41.2 at eight weeks and 40.2 at 20 weeks), after Botulinum injection, those difference were not statistically significant. But it decreased statistically significant when considering audiovisual as a separate item in the presented emotion at 20 weeks after injection (From 50.1 to 46.6) $(\mathrm{P}<0.05)$.

We also analysed the MERT score exclusively in 29 subjects who returned after 20 weeks. By doing that, the changes in the mean MERT score 20 weeks after botulinum injection in the different items like still picture (37.3 to 35.3 ), video (47.2 to 42.7) and audiovisual (50.8 to 46.6) were statistically significant. $(\mathrm{P}<0.05)$ (Fig. 3$)$.

\section{DISCUSSION}

Our study shows that facial emotion recognition probably attenuated by paralysing facial muscle after cosmetic injection of the BTX.

Our results are in accordance with the embodied simulation theory and the role of facial muscle activation in perception and recognition of others emotion. Following mechanisms in the perception and recognition of emotion might be affected by BTX injection:

1. "Facial feedback": This mechanism naturally enhances the subjects own emotional experiences by contracting facial muscles to simulate an emotion without its trigger; Facial feedback is the reason why ancient worriers were constructed to express fearsome expression in the battlefield. By 
The MERT score in different modalities before and 7

and 20 weks after Botulinum injection

60

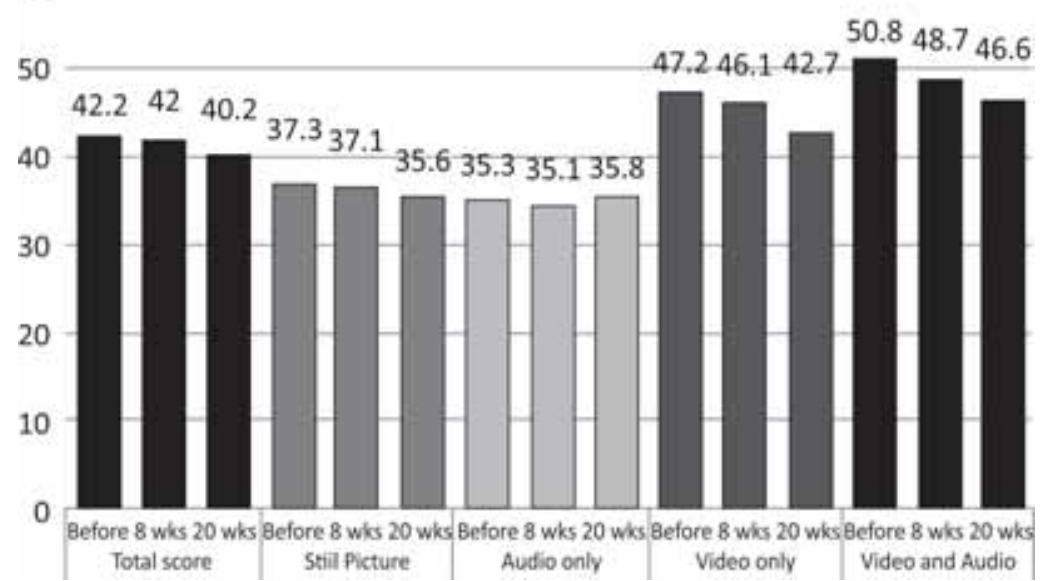

FIGURE 2. The mean MERT score before and 8 and 20 weeks after BTX injection

The MERT score in different modalities before and 8 and 20 weks after Botulinum injection

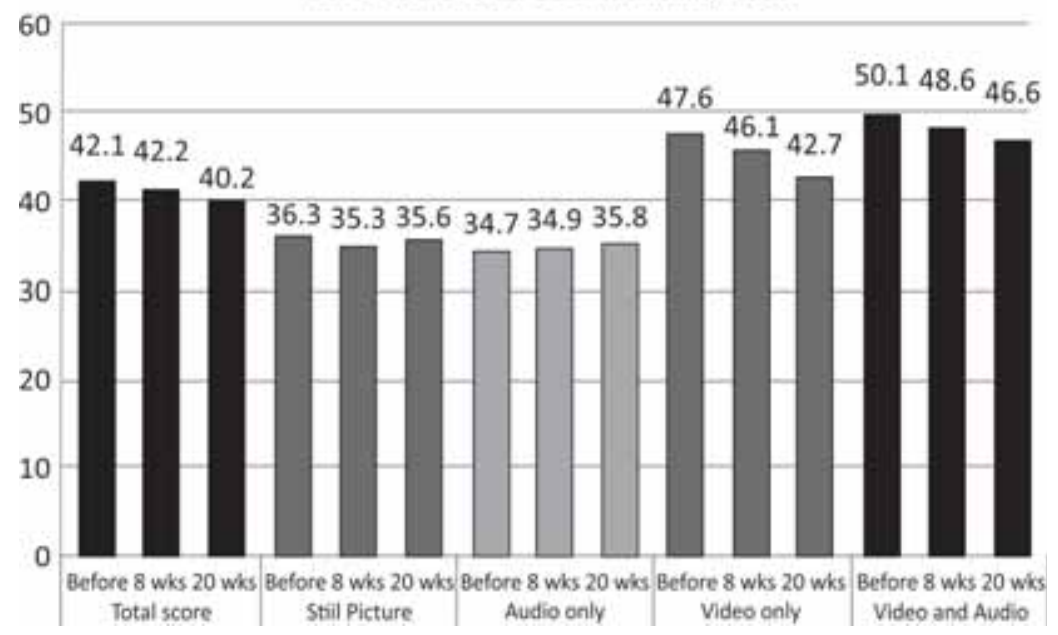

FIGURE 3. The mean MERT score before and 8 and 20 weeks after $B T X$ injection in 29 subjects who complete the evaluation at 20 weeks.

doing this, worriers felt angrier even before confronting the enemy. Charles Darwin put it that way: "even the simulation of an emotion tends to arouse it in our minds" (20). Very first scientific ideas on this subjected were provided by many, to name a few, Gelhorn, Tomkins and Laird introduced facial feedback theory in the early sixties (21-23). Martin and Stepper conducted one of the most interesting studies in 1988. To simulate smiling, they asked subjects to hold a pen between their teeth. The cartoons looked funnier to them compared to normal control (Fig. 4) (24). In recent years there is more evidence that suggests that one's facial changes can affect one's emotional experience (25). Even Fron- tal Electroencephalographic alpha activity declines when remembering pleasant memories $(26,27)$. So after paralysing facial muscle by BTX and reduction of Facial Feedback mechanism, subject feeling about the emotion might be reduced.

2. "Physiological response to emotion": Few studies measured subjects physiological responses after they were instructed to create an artificial emotion without actually feeling it. (For example pulling eyebrows down and together, raise upper eyelid and tighten lower eyelid, narrow lips, and press together to impose anger). Ekman and Levenson observed that the physiological response (Electrodermal or cardiovascular) were similar physio- 


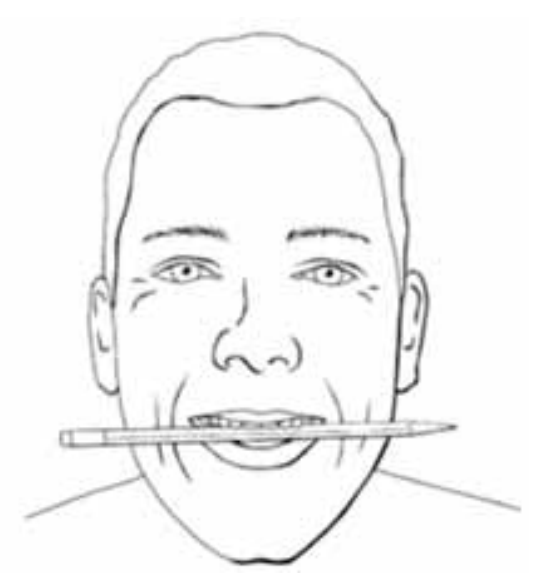

FIGURE 4. When subjects asked to hold a pen between their teeth, the presented cartoons looked funnier to them (21).

logical changes between re-experienced genuine emotions from the past and artificially created facial experiences consistent with such feelings (28). The current view is that facial expressions, not only are the manifestation of internal emotional states, it also can themselves create the physiological changes occurs typically by the experience of the same emotions which might reduce after BTX injection.

3. Reduction of "emotional contagion" mechanism: This is unconsciously expressing the same emotional experiences of others with whom the subjects interact. Schoenewolf defines this phenomenon as "a process in which a person or group influences the emotions or behaviour of another person or group through the conscious or unconscious induction of emotion states and behavioural attitudes" (29). So, After attenuating facial emotional expression, its contagion might also be reduced.

3. Impaired "Facial Mimicry": This is when the same others emotional displays elicit in the index subject.(30) There are pieces of evidence that Hypomimia in Parkinson's disease reduces the ability of recognition negative emotions expressed by others (2). According to Hess and Fischer, facial mimicry is necessary to recognise feeling, when it is hard to be perceived (31).

4. "Changing brain networks". Antonucci et al. argued that BTX may have direct effects on the brain networks via retrograde transport and remote form injection sites in afferent motoneurons (32). It also is shown that amygdala and its reciprocal connections to the limbic system and brain stem response attenuate after BTX injection into facial muscles $(25,33)$.
Needless to say that, the effect of BTX's on facial emotion expression is not so complete because all of the muscle groups involved in any particular emotion could not block from contracting (20).

\section{CONCLUSION}

To conclude, cosmetic injection of BTX into forehead muscles probably reduced recognition of emotions expressed on face.

We provide additional arguments in favor of embodied simulation theory asserting that changing facial emotional expression could alter the recognition of them too.

\section{Limitations}

We lost a high number of our subjects during the 20 weeks follow up. All of the subject who returned were women. Although there might be a gender difference in facial emotion recognition, that might limit our finding to all.

Further randomized and placebo control studies is needed on that subjects.

\section{Author's contribution}

Kaveh Shafiei introduced the idea, wrote the study protocol and the manuscript, revised the statistical analysis. Amir Sherafat was in charge of the logistics for subjects recruitment, BTX injections, examine cosmetic outcome, doing the Multimodal emotion recognition tests, providing study data and statistical analysis. Behnaz Sedighi contributed in writing and discussing the study protocol, study design and manuscript.

\section{Acknowledgment}

We are very grateful to all of all of our subjects for their time in doing the MERT and also appreciate Dr. Mohebi and Dr. Darvish Damavandi who let us use their cosmetic clinic to recruit our subjects, advising on BTX injection procedure, and providing space for our study.

We can not thank enough the Swiss center for affective sciences for their permission on using MERT.

And the last, but not the least the Neurology research center, Kerman University of medical sciences for founding our research. 


\section{REFERENCES}

1. Blair R.J. Facial expressions, their communicatory functions and neuro-cognitive substrates. Philos Trans $R$ Soc Lond B Biol Sci, 2003. 358 (1431): p. 561-72.

2. Heckmann M. et al. Pharmacologic denervation of frown muscles enhances baseline expression of happiness and decreases baseline expression of anger, sadness, and fear. J Am Acad Dermatol, 2003. 49 (2): p. 213-6.

3. Frith $\mathbf{C}$. Role of facial expressions in social interactions. Philos Trans $R$ Soc Lond B Biol Sci, 2009. 364 (1535): p. 3453-8.

4. Wilhelm 0 . et al. Test battery for measuring the perception and recognition of facial expressions of emotion. Front Psychol, 2014. 5: p. 404.

5. Darwin C. The Expression of the Emotions in Man and Animals. Oxford University press, 1872.

6. Argaud S. et al. Does Facial Amimia Impact the Recognition of Facial Emotions? An EMG Study in Parkinson's Disease. PLoS One, 2016. 11 (7): p. e0160329.

7. Davies H. et al. Facial expression to emotional stimuli in nonpsychotic disorders: A systematic review and meta-analysis. Neurosci Biobehav Rev, 2016. 64: p. 252-71.

8. Adolphs R. Recognizing emotion from facial expressions: psychological and neurological mechanisms. Behav Cogn Neurosci Rev, 2002. 1(1): p. 21-62

9. Goldman A.I., Sripada C.S. Simulationist models of face-based emotion recognition. Cognition, 2005. 94(3): p. 193-213.

10. Cortes D.S.L., P. Lindahl, C. Fischer H. Memory for faces and voices varies as a function of sex and expressed emotion, in PLoS One. 2017. p. 19.

11. James W. The prinicple of psychology. New York Holt, 18990.

12. Paul E. Emotion revealed. 2013, New York: Times Book Henry Holt and Company.

13. Ekman P. Facial expression and emotion. Am Psychol, 1993. 48(4): p. 384-92.

14. Lewis M.B., Bowler P.J. Botulinum toxin cosmetic therapy correlates with a more positive mood. J Cosmet Dermatol, 2009. 8(1): p. 24-6.

15. Ian J.S., A. Brandt F. Ochsner K.N. The Effects of BOTOX® Injections on Emotional Experience. Emotion, 2010. 10(3).

16. Magid M. et al. Treating depression with botulinum toxin: a pooled analysis of randomized controlled trials. Pharmacopsychiatry, 2015. 48(6): p. 205-10.

17. Han C. et al. Can botulinum toxin improve mood in depressed patients? Expert Rev Neurother, 2012. 12(9): p. 1049-51.

18. Havas D.A. et al. Cosmetic use of botulinum toxin-a affects processing of emotional language. Psychol Sci, 2010. 21(7): p. 895-900.
19. Banziger T., Grandjean D., Scherer K.R. Emotion recognition from expressions in face, voice, and body: the Multimodal Emotion Recognition Test (MERT). Emotion, 2009. 9(5): p. 691-704.

20. Alam M. et al. Botulinum toxin and the facial feedback hypothesis: can looking better make you feel happier? J Am Acad Dermatol, 2008. 58(6): p. 1061-72.

21. Strack F., Martin L.L., Stepper S. Inhibiting and facilitating conditions of the human smile: a nonobtrusive test of the facial feedback hypothesis. J Pers Soc Psychol, 1988. 54(5): p. 768-77.

22. Ahern G.L., Schwartz G.E. Differential lateralization for positive and negative emotion in the human brain: EEG spectral analysis. Neuropsychologia, 1985. 23(6): p. 745-55.

23. Antonucci F. et al. Long-distance retrograde effects of botulinum neurotoxin A. J Neurosci, 2008. 28(14): p. 3689-96.

24. Ekman P. Facial expressions of emotion: an old controversy and new findings. Philos Trans R Soc Lond B Biol Sci, 1992. 335(1273): p. 63-9.

25. Field T.M., Walden T.A. Production and perception of facial expressions in infancy and early childhood. Adv Child Dev Behav, 1982. 16: p. 169-211.

26. Gellhorn $\mathbf{E}$. The physiological basis of neuromuscular relaxation. AMA Arch Intern Med, 1958. 102(3): p. 392-9.

27. Hennenlotter A. et al. The link between facial feedback and neural activity within central circuitries of emotion - new insights from botulinum toxin-induced denervation of frown muscles. Cereb Cortex, 2009. 19(3): p. 537-42.

28. Hess U., Fischer A. Emotional mimicry as social regulation. Pers Soc Psychol Rev, 2013. 17(2): p. 142-57.

29. Kim M.J. et al. Botulinum toxin-induced facial muscle paralysis affects amygdala responses to the perception of emotional expressions: preliminary findings from an A-B-A design. Biol Mood Anxiety Disord, 2014. 4: p. 11.

30. Laird J.D. Self-attribution of emotion: the effects of expressive behavior on the quality of emotional experience. J Pers Soc Psychol, 1974. 29(4): p. 475-86.

31. Schoenwolf G. Emotional contagion: Behavioral induction in individuals and groups. Modern Psychoanalysis, 1990. 15.

32. SS. T. Affect, imagery, and consciousness. New York: Springer, 1963. 1.

33. Wexler B.E. et al. EEG and EMG responses to emotion-evoking stimuli processed without conscious awareness. Neuropsychologia, 1992. 30(12): p. 1065-79. 